\title{
EDUCACIÓN SUPERIOR: INSTITUCIONALIDAD PARA LOS NUEVOS DESAFÍOS
}

\section{Introducción}

A través de los últimos años se ha prestado creciente atención a los nuevos desafíos para la educación superior, especialmente en lo que se refiere al aporte que ésta puede hacer para dar un nuevo impulso al desarrollo del país. Adicionalmente, aspectos substantivos de estos desafíos y de su alcance gozan de un amplio grado de acuerdo, lo que se aprecia al revisar los análisis de los diversos especialistas nacionales así como los informes de los organismos especializados -nacionales e internacionales- que han estudiado esta materia.

El conjunto de propuestas elaboradas por el Consejo Nacional de Innovación para el periodo 2010-2020 se basa en que para alcanzar el desarrollo Chile tiene que dar el salto hacia una sociedad del conocimiento, dentro de la cual la educación superior tiene una responsabilidad central, desde la formación de personas que respondan a las competencias que necesitan los diversos sectores productivos, pasando por su aporte en la transferencia de conocimiento a las empresas, hasta la generación de nuevo conocimiento útil a las distintas esferas de la sociedad. Ideas similares respecto al papel de la educación terciaria se encuentran en los informes elaborados por el Consejo Asesor Presidencial para la Educación Superior (2008), el Ministerio de Educación (2009), la Organización para la Cooperación y el Desarrollo Económicos junto al Banco Mundial (2009). En ellos se resalta que en la actualidad el rol de la educación superior debe orientarse a generar las capacidades para que los diversos actores sociales sean protagonistas del progreso del país, en el sentido de transitar desde una economía que ha logrado altos niveles de eficiencia y requiere ahora pasar a una etapa caracterizada por la innovación. Esto significa formar a personas que puedan participar en todos los aspectos de la vida social -desde el trabajo hasta los procesos de decisión ciudadana-, es decir, que tengan las habilidades para desempeñarse 
en empresas que enfrentan la presión de incorporar conocimientos e innovaciones relevantes en las actividades productivas, aportando también a que los gobiernos mejoren sus políticas, la convivencia y el funcionamiento de los procesos democráticos.

Sin embargo, este evidente acuerdo en torno al tipo de metas que hay que alcanzar no es garantía suficiente para que el sistema sea capaz de cumplirlas, ante lo cual surge la pregunta de si el actual diseño institucional de la educación terciaria -cuyo origen se remonta a 1981- tiene los incentivos necesarios para enfrentar con éxito los desafíos que hoy se le imponen o llegó el momento de su revisión. Para responder esta interrogante se deben revisar los desafíos de la educación superior en Chile, observar que el sistema tiene múltiples objetivos que están interrelacionados entre sí y que involucran a muchos grupos de actores sociales, como los estudiantes, los docentes, las empresas, los directivos de las instituciones educacionales y la sociedad en su conjunto. En este contexto, un desafío inevitable es revisar el marco institucional que moldea las reglas del juego con que operan estos actores, de modo que en sus interacciones logre compatibilizar sus intereses particulares con los objetivos del interés social.

\section{Los desafíos de la educación superior}

Desde mediados del siglo pasado el país ha transitado desde un sistema de estudios superiores elitista y poco diversificado, financiado principalmente por el Estado, hacia uno masivo y diversificado, que se financia mayoritariamente con recursos privados. Esta transición se inicia en la década del 1960, cuando se acelera el crecimiento de la demanda por educación superior, lo que significó un incremento en el financiamiento público. En esos años la institucionalidad tenía dificultades para satisfacer los nuevos requerimientos de cobertura y de acceso, por lo que se llegó a la reforma de 1981, la que autorizó la creación de universidades privadas e instituciones no universitarias (institutos profesionales y centros de formación técnica) con el objetivo de expandir la cobertura, estimular la competencia y diversificar la oferta ${ }^{2}$. Esta reforma fue acompañada por un enfoque de regulación

2 Bernasconi y Rojas (2004) ofrecen una revisión detallada de esta reforma. 
mínima que confiaba en el funcionamiento del mercado e impedía a la autoridad orientar el desarrollo del sector.

En las tres décadas siguientes dicha institucionalidad ha tenido pocos ajustes. Se pueden mencionar la creación del Consejo Superior de Educación en 1990; el Programa de Mejoramiento de la Calidad y la Equidad de la Educación superior (MECESUP) de 1998 y la Ley 20.129 que establece un Sistema Nacional de Aseguramiento de la Calidad de la Educación Superior el año 2006.

Así se llegó al sistema educativo que tenemos en la actualidad, el que se caracteriza por un alto grado de autonomía de las instituciones que lo componen y se expresa en una amplia libertad en la generación y en la gestión de productos. Adicionalmente, se ha creado una gran diversidad institucional coordinada a través de mecanismos de mercado. Tal y como sostiene José Joaquín Brunner en su libro "Educación Superior en Chile", la estructura del mercado de la educación superior del país se caracteriza por un amplio predominio de la oferta privada, a lo que agrega que "desde el punto de vista del análisis económico la existencia de mercados en la educación terciaria depende del grado en que se cumplen las condiciones (o "libertades") para su funcionamiento, tanto para los proveedores (las "firmas" o agentes institucionales) como para los consumidores (estudiantes)." (Brunner, 2009; p. 431).

Bajo este marco institucional los estudios e informes mencionados anteriormente formulan un conjunto de desafíos específicos para que la educación superior pueda cumplir la misión de aportar al desarrollo del país en la fase actual. Para los fines de este ensayo estos retos se agrupan en cinco áreas:

\section{Reorganización de los productos}

Existe un acuerdo amplio en que la educación superior debe reorganizar la arquitectura de los productos que entrega a la sociedad, incluyendo la articulación eficiente entre los diferentes subsistemas del sector, la adopción de un mecanismo que facilite la formación continua y la mayor sintonía de los estudios con los requerimientos de las actividades productivas. Según estos informes se debe adoptar 
un régimen de educación superior más flexible y con una mejor coordinación de la educación de pre y posgrado, así como entre los estudios técnicos y universitarios.

También se plantea que debería eliminarse el vínculo entre grados profesionales y académicos, como es el requisito legal de tener una licenciatura antes de obtener algunos grados profesionales. Este cambio significaría eliminar la lista cerrada de grados profesionales universitarios definida por ley, los que tienen como requisito obtener una licenciatura y que sólo pueden ser otorgados por universidades. Estas últimas junto a los institutos profesionales deberían tener la libertad de brindar los grados que fueran capaces de ofrecer en un contexto amplio de tres ciclos, similar al del proceso de Bolonia (Armanet, 2004).

De acuerdo con la tendencia mundial, se tendría que pasar a programas de primer grado más cortos, es decir, habría que revisar la carga académica de los programas de las instituciones de educación superior, teniendo en cuenta tanto las necesidades nacionales como los estándares internacionales, para desarrollar currículos menos recargados y más eficientes, así como también establecer una mayor articulación con los niveles anteriores de educación, de modo de reducir la brecha de conocimiento necesario para el ingreso a la educación terciaria y disminuir la alta tasa de deserción que en la actualidad tienen las universidades. Esta revisión busca introducir mayor flexibilidad, regular los grados de especialización e introducir elementos curriculares adicionales tales como trabajo en equipo, destrezas comunicacionales, conciencia intercultural, espíritu empresarial y aprendizaje de un segundo idioma con un alto nivel de competencia.

Otro aspecto sugerido en esta reorganización es la adopción de un enfoque de competencias en la educación postsecundaria, especialmente en la técnica, en la forma de un Sistema Nacional de Certificación de Competencias Laborales, como lo señala el Informe del Consejo Nacional de Innovación para la Competitividad (2010). Estas competencias son aquellas habilidades que deben adquirir las personas tras cursar diferentes programas y niveles. Ello supone la 
certificación de los resultados del aprendizaje, lo que está en la base de la acreditación de competencias laborales. Este cambio precisa que los sectores productivos participen en la definición de las competencias laborales que orientan los procesos formativos.

Los cambios en la arquitectura de los productos del sector debieran llevar a una homologación de los módulos de formación, de modo de facilitar la movilidad de los estudiantes. Esto demanda la armonización de los procesos de formación entre las diferentes instituciones.

Otro aspecto de la reorganización de los productos de la educación superior consiste en adaptar el sistema para que opere eficientemente a lo largo de la vida. Existe alta coincidencia entre los distintos informes que analizan la educación superior chilena, en cuanto a que se debe avanzar hacia un sistema de aprendizaje continuo, accesible y de calidad internacional, el que además tiene que ser flexible en el sentido de permitir a cada persona trazar su itinerario de formación.

La experiencia internacional muestra que para la implementación de un sistema de este tipo han resultado esenciales la coordinación público-privada y la participación del sector productivo -a través de consejos sectoriales- en el desarrollo y actualización de estándares que permitan que la formación técnica y la capacitación respondan efectivamente a las demandas presentes y esperadas del mercado laboral. Por otra parte, el informe OCDE-Banco Mundial (2009) expresa que se debería desarrollar un Marco de Cualificaciones Nacionales, que abarque todas las calificaciones, académicas y vocacionales, desde la educación secundaria hasta el doctorado.

\section{Mejorar la información}

El segundo reto es mejorar la información en las decisiones de estudiantes y empleadores. El sistema de educación superior presenta aún altas asimetrías de información, incluyendo variables como empleabilidad, calidad de los estudios y tasas de deserción. 
Un paso importante para reducir esta brecha fue el sistema de acreditación, pero se trata de un avance aún insuficiente. Los actuales criterios de acreditación han sido evaluados como demasiado vagos debido a que dejan un amplio margen para la interpretación de los evaluadores y no están lo suficientemente adaptados a la variedad de instituciones y a sus diferentes misiones. La actual acreditación institucional es voluntaria y se debería lograr que todas las instituciones la soliciten, de modo de incentivar la calidad. A su vez, cuando la mayoría de las instituciones del sistema la hayan obtenido será necesario establecer niveles de referencia más estrictos.

El paso siguiente es asegurar que la información estimule la innovación y el mejoramiento continuo de la enseñanza, el aprendizaje, la investigación académica y las actividades de extensión. Una experiencia relevante es la información que aporta la iniciativa Futuro Laboral del Sistema Nacional de Información de la Educación Superior, lo que se puede profundizar con una interpretación más completa de los datos del mercado laboral para los estudiantes, sus familias y profesionales especializados. Según el informe de la OCDEBanco Mundial, el país debería establecer un sistema de información unificada sobre la calidad de los programas académicos, la tasa de deserción de los alumnos y sus causas. Con el tiempo, esta base de información tendría que extenderse para incluir encuestas sistemáticas de seguimiento de graduados y estudios sobre la satisfacción de los empleadores con respecto a los titulados.

\section{Un sistema integrado de financiamiento}

A partir de las recomendaciones de la OCDE-Banco Mundial sería necesario que el MINEDUC fusione los programas actuales de becas en uno solo, con un pequeño número de "ventanas", y que se unan también los dos programas de crédito universitario que existen. Más que el modelo del Fondo Solidario sería preferible fusionar los dos programas de préstamos en uno similar al crédito con aval del Estado. $^{3}$

3 Los trabajos que se reúnen en Bernasconi (ed.) (2002) ofrecen una discusión interesante al respecto. 
Podría ser más eficiente y efectivo tener menos instrumentos de financiamiento, pero más amplios y mejor enfocados. Habría también que explorar las modalidades de convenios entre el Estado y las instituciones de educación superior para enseñar programas con matrícula garantizada. Hoy existen demasiados instrumentos de financiamiento, con bastante duplicación, para promover vínculos industria-universidad, becas para estudios de doctorado y apoyo a centros de investigación; a lo que se agrega que algunos de los que existen son demasiado pequeños. Las dificultades en este ámbito son de economía política, lo que requiere de una visión compartida de los actores.

\section{Provisión de bienes públicos}

El Consejo Nacional de Innovación para la Competitividad recomienda que el aporte basal a las universidades esté fundado en la definición y reconocimiento de aquellos bienes públicos o servicios que la sociedad demanda a través del Estado. Este financiamiento debe estar supeditado a la existencia de externalidades positivas asociadas a la generación de masas críticas de profesionales de excelencia, principalmente en regiones, o a ciertos beneficios sociales.

Según las recomendaciones dadas por la OCDE-Banco Mundial, para ayudar a lograr un financiamiento de infraestructura más estable se tendría que identificar un número potencial de universidades enfocadas a la investigación, algunas de las cuales podrían perfectamente privilegiar las prioridades regionales, lo que significa cambios en los modelos de financiamiento existentes. Las instituciones de educación superior con asesoría del gobierno tendrían que decidir hacía dónde quieren dirigirse. Aquellas que no tengan la intención de obtener un estatus de universidad de investigación, podrían desear entregar educación de pregrado de alta calidad, o limitar sus actividades de investigación a unas pocas áreas de relevancia regional, o bien, poner énfasis en temas especiales tales como la tecnología. Lo anterior permitiría, además, entregar incentivos para aumentar los lazos con el área de negocios y la industria. Desafortunadamente, hoy los incentivos llevan a que todas las universidades traten de hacer 
lo mismo, impidiendo la especialización y la generación de masas críticas en investigación.

Por otra parte, entre las recomendaciones del Consejo Asesor se encuentra que el Estado defina un conjunto de criterios que permitan identificar aquellas instituciones estatales que, por razones que el Estado debe explicitar, requieren apoyos específicos, determinando las condiciones a las que ellas deben subordinarse para obtenerlos.

\section{Integración al mundo}

La internacionalización de la educación superior ha sido identificada como una prioridad importante por el gobierno chileno. Sin embargo, el equipo revisor de la OCDE-Banco Mundial consideró que no hay un plan nacional claro para lograrlo y que el progreso, hasta la fecha, ha sido irregular.

Estos desafíos no son recientes, han estado presentes en los diversos análisis de la educación superior en la última década. Por esta razón surge la inquietud de si el actual sistema tiene las capacidades para asumir y enfrentar con éxito las transformaciones necesarias. La respuesta a esta interrogante hace imprescindible el análisis de su gobernabilidad.

\section{El modelo de gobernabilidad}

De acuerdo al esquema tradicional de la organización industrial, el desempeño de los sectores económicos depende de la conducta de los actores, la que a su vez está explicada por la estructura de incentivos que éstos enfrentan. En el caso de la educación superior la gobernabilidad corresponde al conjunto de mecanismos o reglas del juego que regulan las transacciones de los productos que ofrecen las instituciones y establecen los incentivos correspondientes. Estos mecanismos, que pueden ser legales, de regulación sectorial o de normas sociales, causan el efecto de facilitar o restringir las opciones que tienen los participantes de estas transacciones, incluyendo los sistemas que permiten asegurar el cumplimiento de los compromisos y contratos. 
La función que cumple el modelo de gobernabilidad es proteger los intereses de cada una de las partes involucradas en los intercambios sociales que ocurren en este sector y compatibilizar estos intereses con los de la sociedad en su conjunto. Todo ello, en un ambiente de importantes externalidades y de asimetrías de información.

Las dos variables que caracterizan el modelo de gobernabilidad son la disciplina de la competencia del mercado y la disciplina que produce el gobierno. En la mayoría de los mercados de bienes y servicios la primera es una condición necesaria y suficiente para asegurar que las interacciones descentralizadas estén alineadas con el interés social, por lo que resulta innecesaria una acción complementaria del gobierno. Sin embargo, hay muchos mercados de bienes y servicios que no conducen a un resultado eficiente y es indispensable la acción pública.

Tomando en cuenta las combinaciones entre estas dos variables se pueden definir cuatro modelos de gobernabilidad, los que se presentan en la tabla 1:

- Cuadrante A: existe una regulación fuerte del gobierno y una escasa disciplina de mercado. En esta situación están los bienes públicos y los monopolios naturales.

- Cuadrante B: la regulación es débil y también hay una limitada disciplina del mercado porque existe poca competencia, debido a que se presentan muchas asimetrías de información o bien hay abundantes externalidades.

- Cuadrante C: hay gran descentralización de actores que pueden contratar los productos con la disciplina de la competencia. Este modelo funciona bien cuando los mercados tienen fallas de poca significación.

- Cuadrante D: hay una acción descentralizada de los actores que se combina con una acción reguladora del gobierno. Entre ambas puede existir cierta tensión, pero los dos aspectos son indispensables para un resultado eficiente. Este es el caso del sistema financiero en el que existen importantes asimetrías de información y una activa regulación. 
Tabla 1: Modelos de gobernabilidad

\begin{tabular}{|c|c|c|c|}
\cline { 3 - 4 } \multicolumn{2}{c|}{} & \multicolumn{2}{c|}{ GOBIERNO } \\
\cline { 3 - 4 } \multicolumn{2}{c|}{} & Activo & Pasivo \\
\hline \multirow{2}{*}{ MERCADO } & Limitado & - A - & - B - \\
\cline { 2 - 4 } & Extendido & - D - & - C - \\
\hline
\end{tabular}

Dentro de cada uno de estos modelos hay matices y variaciones, por lo que en diversos casos los cambios en los esquemas de gobernabilidad deben ser analizados en términos de dirección más que como saltos de un cuadrante a otro. Además, cada sociedad cuenta con un conjunto de instituciones que dan soporte al funcionamiento de las diversas actividades económicas y que influyen en el funcionamiento de los mercados, por lo que los mecanismos que funcionan bien en algunos países podrían generar resultados muy diferentes en otros. Por esta razón es indispensable evaluar el funcionamiento de los modelos de gobernabilidad en su propio contexto ${ }^{4}$.

En las primeras fases del desarrollo de la educación superior en Chile se aplicaba un modelo de gobernabilidad del tipo descrito en el cuadrante B, con pocas instituciones en el lado de la oferta y un rol limitado del gobierno. Bernasconi (2002) plantea que este sistema ha sido frecuente en América Latina por los privilegios asociados a la autonomía que tienen las universidades respecto del Estado.

Con la reforma de 1981 se aplicó en Chile un modelo como el descrito en el cuadrante $C$, que aumentó considerablemente el número de instituciones, otorgó bastante libertad en la gestión de los productos y mantuvo una limitada presencia del gobierno. Considerando el objetivo de ampliar la cobertura del sector superior, este modelo fue exitoso. Sin embargo, su resultado ha sido un sistema con una integración insuficiente, con incentivos débiles para mejorar en calidad, sin una visión común de sus desafíos y con tenues mecanismos de cooperación. Por ejemplo, un tercio del gasto público en educación superior no es concursable ni está sujeto a criterios de desempeño.

4 La literatura especializada tiende a asociar el modelo con gobierno activo al sistema europeo y con un gobierno pasivo al sistema norteamericano, sin embargo este análisis omite otras variables sistémicas que influyen en el desempeño del sistema en cada caso. Ver Brunner (2009). 
En la actualidad el principal problema es que el modelo de gobernabilidad que combina una fuerte descentralización de las instituciones de educación superior con una débil acción del gobierno no permite enfrentar los nuevos desafíos del sistema. Ante esta realidad autores como Levy (2002) han planteado que las propias instituciones universitarias deben actuar con mayor responsabilidad social, pero este requerimiento tiene implícita una base institucional y de capital social que no existe en Chile. Desde que se aplica este modelo, la formación terciaria ha mantenido un alto crecimiento, no obstante, el esquema descentralizado puede generar una sobreextensión en el número de establecimientos, especialmente en segmentos de menor calidad. Es decir, un paso indispensable para enfrentar la nueva agenda de la educación terciaria es encontrar un nuevo balance en la gobernabilidad del sector. Solo de esta manera se puede incrementar el aporte de la educación al desarrollo.

La primera razón por la que el actual esquema de gobernabilidad de la educación postsecundaria no permite enfrentar los nuevos desafíos es que lo que se necesita es generar una serie de activos públicos, que benefician a todos los actores. Entre ellos está la articulación entre las partes, lo que permite aprovechar complementariedades y otorga flexibilidad a los estudiantes. Sin embargo, lo que beneficia a los alumnos puede significar un perjuicio para algunas instituciones educacionales, así algo que es socialmente deseable se puede bloquear en un sistema descentralizado que tiene costos de coordinación.

Cuando se amplían las posibilidades de elección por parte de los estudiantes se les concede un mayor protagonismo, lo que generaría una desventaja en muchas instituciones porque necesitarían ajustar sus estrategias y programas y, además, quedarían más expuestas a las decisiones de los alumnos, las que en un sistema modular producirían mayor movilidad y competencia.

Por ello, un sistema integrado y articulado en sus diversos componentes es un activo público. Ninguna institución por sí sola querrá hacer los ajustes, quedando vulnerable ante un escrutinio más exigente de los estudiantes. La movilidad y la integración de los distintos subsistemas de la educación superior benefician directamente 
a quienes se educan, pero tiene un efecto incierto en las instituciones educacionales. Esta es la razón que hace que la reorganización del sector no sea posible con el actual sistema de gobernabilidad -de instituciones descentralizadas y de un gobierno débil-. La única opción es un esfuerzo colectivo para colocar incentivos más poderosos. Incluso si se organiza el sistema en forma coordinada algunas instituciones se van a ver beneficiadas y otras perjudicadas. Por esta razón, el actual modelo de gobernabilidad descentralizado logrará avances insuficientes en la creación de este tipo de activos.

La segunda causa que explica la ineficiencia del actual modelo de gobernabilidad es que hay asimetrías de información que las instituciones pueden utilizar en su beneficio. El sistema de acreditación que buscaba reducir dichas asimetrías de información dejó las cosas a medio camino, con avances importantes pero con incentivos que tienen menos fuerza que la necesaria para que la inversión en calidad de las instituciones genere mayores beneficios.

El sistema de información también es un bien público, por lo que es difícil que los establecimientos quieran aportar información sin la coordinación de la autoridad. También el sistema de información tiene costos de producción y su beneficio depende de la calidad de la información y de la posibilidad de que sea verificada. La información que las universidades están dispuestas a entregar en el sistema actual es inferior a la que se precisa en condiciones óptimas. Éste tiene un incentivo a sobre invertir en publicidad o en imagen de marca y a subinvertir en factores que influyen directamente en la calidad producida.

Otra debilidad del modelo vigente se manifiesta en la capacidad de las instituciones de educación superior para producir bienes públicos. El esquema descentralizado funciona bien en conjunto con concursos de proyectos de investigación o de infraestructura de montos medianos, pero tiene dificultades como sistema para orientar inversiones de montos superiores o con un ciclo de maduración de largo plazo. Se precisa un sistema de contratos de desempeño y de vigilancia de los mismos, incluyendo financiamiento basal. Los nuevos desafíos necesitan de sistemas de evaluación del desempeño que sean 
transparentes y confiables, en caso contrario, lo que podría ocurrir es un aumento del aporte público con poca efectividad en resultados.

Vincular financiamiento con desempeño es un requisito clave para producir eficientemente bienes públicos en forma descentralizada (Bernasconi, 2002). Pero esta estrategia requiere de un mecanismo que mida el desempeño en forma confiable, para lo que necesita contar con capacidad institucional, lo que no es posible en el sistema actual. La consecuencia es desaprovechar el potencial de las instituciones de educación superior para aportar bienes públicos que el país necesita.

La tercera razón apunta a que existen costos de coordinación cuando las instituciones quieren innovar en sus productos. Por ejemplo, cuando se propone adaptar los productos para el aprendizaje a través de la vida la incertidumbre del cambio le genera un costo mayor a las primeras instituciones que deciden moverse hacia el nuevo sistema. Lo mismo ocurre con la articulación entre subsistemas, cuyos beneficios dependen de las estrategias que adopten las demás instituciones. Si todos lo hacen, los beneficios son altos, pero si sólo una modifica sus productos logrará beneficios mínimos. Asimismo, cuando varios de los desafíos implican incorporar los aportes del sector productivo, las necesidades de coordinación se hacen más complejas. Por ejemplo, en relación al sistema de competencias, su implementación no es posible sin participación de todos los sectores involucrados, incluyendo al productivo. Por ello los cambios necesitan de una acción coordinada y que el esquema descentralizado hace que los cambios indispensables sean más lentos.

También hay muchas necesidades de coordinación entre el sistema de educación superior y el sector productivo. Esta articulación tiene un interés social, por lo que necesita de la intervención del gobierno para que ocurra. Los nuevos desafíos necesitan construir lazos más estrechos entre los requerimientos de los empleadores y los programas académicos de las instituciones de educación terciaria, los que tendrían que incluir la participación y el compromiso de ambas partes. 
En cuarto lugar, en la medida que aumenta la cobertura de la educación superior el crecimiento del sector debe considerar más los aspectos de calidad que de cantidad. Esto es relevante en las normas de instalación de nuevos establecimientos o de nuevas sedes que generan un desplazamiento de la demanda desde los ya existentes a los nuevos. En condiciones de información imperfecta el efecto agregado puede reducir la calidad promedio y aumentar los costos unitarios de los establecimientos existentes. El número de establecimientos de baja calidad que genera el sistema descentralizado es mayor que lo que se esperaría en forma óptima.

En todos estos casos hay una brecha entre el interés privado y el interés social, lo que solo es posible de resolver mediante un nuevo balance entre la disciplina de mercado y la intervención del gobierno.

\section{Conclusiones}

La conclusión del análisis anterior es que el modelo de gobernabilidad de la reforma de 1981, que ha tenido pocas modificaciones en las décadas siguientes, no tiene las capacidades para enfrentar con éxito los nuevos desafíos de la educación superior. Es posible hacer avances parciales de diversos ámbitos, pero ninguno de estos cambios logrará aprovechar todo el potencial que tiene el aporte de la educación superior al desarrollo del país. Los cambios en la arquitectura de los productos o en su calidad no surgirán de forma espontánea con el actual modelo de gobernabilidad.

La estrategia del cambio debe considerar que la regulación de la educación superior tiene un costo, por lo que se debe indagar en el fortalecimiento de los incentivos hasta el punto en que el sistema se mueva en la dirección deseada, pero evitando generar una regulación que limite la competencia o la innovación de las instituciones.

Para hacer esta indagación es importante generar una visión compartida del punto de llegada hacia el cual se orientan los incentivos. Los nuevos desafíos y la experiencia internacional están contenidos en un conjunto de informes especializados, pero todavía 
no están presentes en las estrategias de las instituciones o del gobierno. Una visión compartida necesita de nuevos liderazgos en el gobierno y en las instituciones educacionales, que ayuden a generar una visión de futuro que permita identificar los objetivos que debemos alcanzar. Una visión de largo plazo requiere de altos niveles de confianza, los que debemos construir. La formación de sindicatos de universidades no hace ningún aporte a generar esta visión compartida, además, es necesario incorporar a las otras partes interesadas.

Por otra parte, la visibilidad de la estrategia precisa definir un conjunto de incentivos que aseguren que las acciones de cada una de las instituciones y de los demás actores relevantes están alineadas. El actual sistema tiene una descentralización excesiva, que hace difícil trabajar con objetivos de interés social.

El nuevo modelo de gobernabilidad debe tener una descentralización balanceada con responsabilidades hacia los objetivos sociales. Esto significa un sistema más estricto de acreditación; que ésta tenga consecuencias visibles en las decisiones estratégicas de las instituciones; mecanismos de coordinación con los sectores productivos; sistemas más sofisticados de información; contratos más eficientes para la provisión de bienes públicos; financiamiento de convenios de desempeño plurianuales para mejorar el acceso, la permanencia y la titulación de estudiantes; y normas de transparencia y rendición de cuentas en la utilización de los recursos públicos.

Estas reformas requieren fortalecer la capacidad institucional del sector público de conducir el proceso, incluyendo una separación de las funciones de diseño de las políticas, de los organismos encargados de su implementación. La gestión de las instituciones se debe mantener descentralizada y flexible, dejando a éstas sujetas a reglas del juego uniformes y a una sana competencia.

Se agradece la valiosa colaboración de Carolina Salazar y Daniela Crovetto en la preparación de este ensayo, así como las agudas precisiones de dos árbitros anónimos. 


\section{Referencias bibliográficas}

Armanet, P. (2004) "Formación universitaria europea. Un ejemplo para Chile". Serie En Foco No 9, Expansiva.

Bernasconi, A. (ed.) (2002) "La Educación Superior ante el Derecho". Editorial Biblioteca Americana. Santiago de Chile.

Bernasconi A.; Rojas, F. (2004) "Informe sobre la Educación Superior en Chile". Editorial Universitaria, Santiago de Chile.

Brunner, J.J. (2009) "Educación Superior en Chile: Instituciones, Mercados y Políticas Gubernamentales", Ediciones Universidad Diego Portales, Santiago de Chile.

Consejo Nacional de Innovación para la Competitividad (2010) "Agenda de Innovación y Competitividad 2010-2010”, disponible en www.cnic.cl

Ministerio de Educación (2009) "Bases para una Política de Formación TécnicoProfesional en Chile, Informe Ejecutivo", Santiago de Chile.

Levy, D. (2002) "La nueva responsabilidad social en la educación superior: repercusiones en el Estado y el Derecho. En Bernasconi, A. (ed.) "La Educación Superior ante el Derecho”. Editorial Biblioteca Americana. Santiago de Chile, 2002.

OCDE-Banco Mundial (2009) "Educación Superior en Chile 2009", disponible en http://bligoo.com/media/users/3/181209/files/18813/EdSupOCDEBM_CNIC.pdf. 